\title{
Infrared Light Curves and the Detectability of Close-In Extrasolar Giant Planets
}

\author{
L. Jeremy Richardson ${ }^{1}$, Sara Seager ${ }^{2}$, Drake Deming ${ }^{3}$, Joseph \\ Harrington $^{4}$, Richard K. Barry ${ }^{1}$, Jayadev Rajagopal ${ }^{1}$, \\ and William C. Danchi ${ }^{1}$ \\ ${ }^{1}$ Exoplanets and Stellar Astrophysics Laboratory, NASA Goddard Space Flight Center, \\ Mail Code 667, Greenbelt, MD 20771, USA \\ email: richardsonlj@milkyway.gsfc.nasa.gov \\ ${ }^{2}$ Department of Terrestrial Magnetism, Carnegie Institution of Washingtone \\ 5241 Broad Branch Rd. NW, Washington, DC 20015, USA \\ ${ }^{3}$ Planetary Systems Laboratory, NASA Goddard Space Flight Center, \\ Mail Code 693, Greenbelt, MD 20771, USA \\ ${ }^{4}$ Cornell University, 326 Space Sciences Bldg., Ithaca, NY 14853, USA
}

\begin{abstract}
We compute theoretical infrared light curves for several known extrasolar planets. We have constructed a set of routines to calculate the orbital parameters for a given planet and integrate over the planetary disk to determine the total flux density of the planet as it orbits the parent star. We have further developed a spectral synthesis routine to calculate theoretical spectra of extrasolar giant planets from 3-24 $\mu \mathrm{m}$. The code requires a temperaturepressure profile as input, calculated by solving the radiative transfer equation; it then calculates continuous opacities and line opacities for water, carbon monoxide, and methane, and finally integrates over the layers of the atmosphere to determine the emergent flux. By integrating the theoretical spectrum over the bandpass of a particular instrument and including realistic instrument noise, we produce a set of multi-wavelength, infrared light curves. Using these light curves, we predict whether a particular known planet can be observed and characterized using the Spitzer Space Telescope, as well as other proposed space-based instruments, such as the Fourier-Kelvin Stellar Interferometer (FKSI) and the James Webb Space Telescope (JWST).
\end{abstract}

Keywords. methods: analytical; stars: individual (HD 209458).

\section{Theoretical Light Curves}

The following is a brief outline of steps in computing the theoretical light curve (implemented in IDL):

- Read the physical parameters for the star and planet.

- Calculate the orbital elements for the planetary system from spherical trigonometry considerations.

- Treat the planet as a blackbody and assume a non-zero temperature asymmetry between the day and night sides of the planet.

- Integrate over the visible portion of the planetary disk as a function of its orbital position. This gives the emitted radiation of the planet as a function of time.

Example theoretical calculations for HD $209458 \mathrm{~b}$ and 55 Cancri b at $24 \mu \mathrm{m}$ are shown in Figures 1 and 2, respectively. We estimate the photon noise due to the planet and star for the known bandwidth and given integration time $(\mathrm{t}=10 \mathrm{sec})$ for Spitzer $(85 \mathrm{~cm}$ aperture) at the MIPS wavelength of $24 \mu \mathrm{m}$ Rieke et al., 2004. The primary eclipse (when the planet transits the star) and the secondary eclipse (when the planet disappears behind the star) are shown for the case of HD $209458 \mathrm{~b}$. 


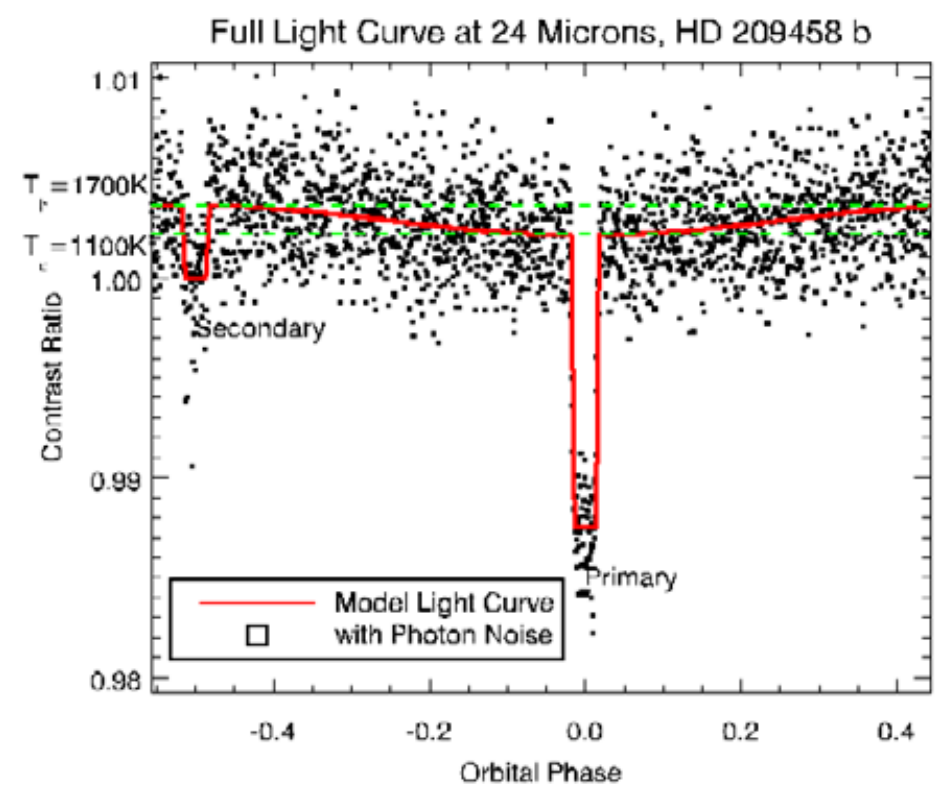

Figure 1. Theoretical light curve for HD $209458 \mathrm{~b}$ with photon noise included for Spitzer/MIPS. We adopted $\mathrm{R}_{*}=1.146 \mathrm{R}_{\text {sun }}, \mathrm{R}_{p}=1.347 \mathrm{R}_{J}, i=86.68$ degrees, $\mathrm{M}_{*}=1.107 \mathrm{M}_{\text {sun }}$ Brown et al., 2001, and eccentricity $\epsilon=0.0$ Deming et al., 2005. The dashed lines indicate the assumed temperature asymmetry of $600 \mathrm{~K}$.

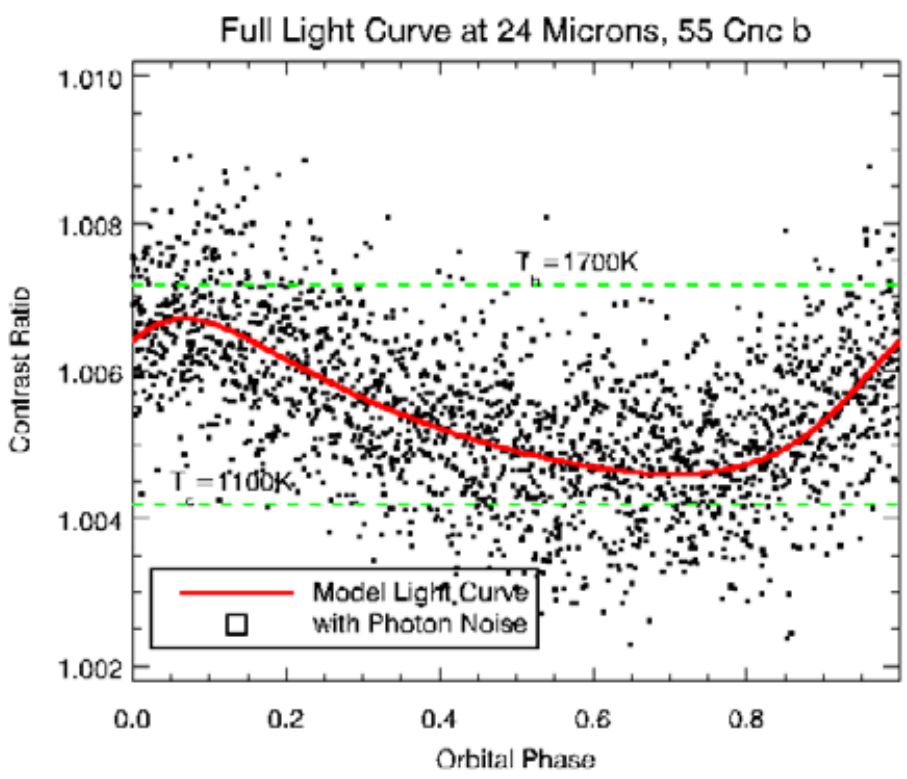

Figure 2. Theoretical light curve for $55 \mathrm{Cncb}$ with photon noise included (also for Spitzer/MIPS). We adopted $\mathrm{R}_{*}=0.87 \mathrm{R}_{\text {sun }}$ Butler et al., 1997 and assumed $\mathrm{M}_{*}=1.107 \mathrm{M}_{\text {sun }}$, $i=45$ degrees, $\mathrm{R}_{p}=1.347 \mathrm{R}_{J}$, and $\epsilon=0.03$, which was increased to show the effect on the light curve. Again, the dashed lines indicate the assumed temperature asymmetry of $600 \mathrm{~K}$.

We further compare the theoretical light curves to actual data on the eclipse of HD 209458 b. Figure 3 shows that we can match the Spitzer/MIPS data on the primary 


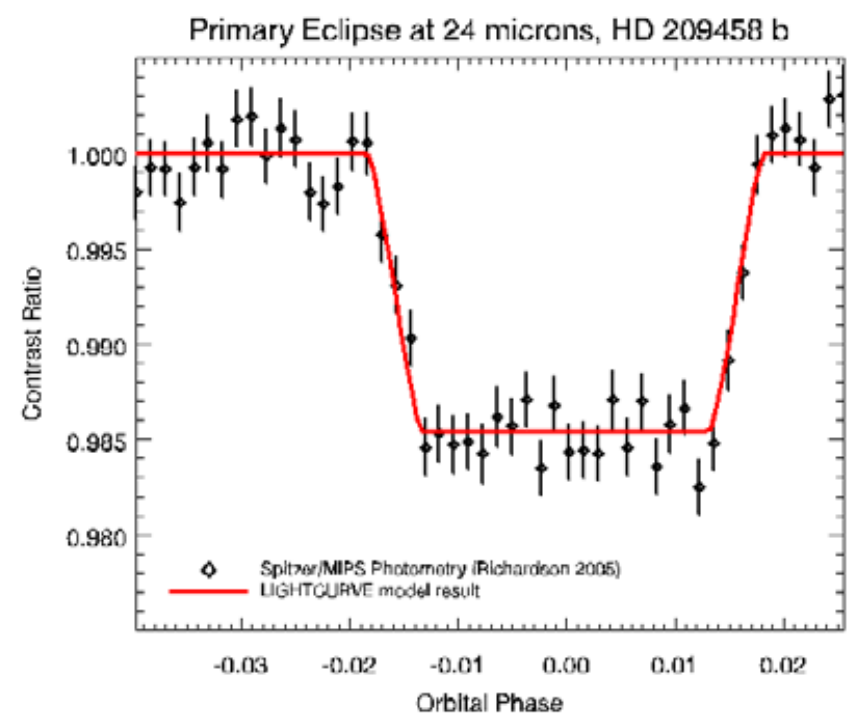

Figure 3. Actual data on the primary eclipse of HD $209458 \mathrm{~b}$ at $24 \mu \mathrm{m}$ from Spitzer, averaged in phase bins of width 0.002 . Theoretical light curve (solid line) was calculated for $\mathrm{R}_{p}=1.29 \mathrm{R}_{J}$ at $24 \mu \mathrm{m}$, and $\chi_{\nu}^{2}=1.035$.

eclipse at $24 \mu \mathrm{m}$. Our routine can also reproduce the now-famous HST curvet of the eclipse in the visible Brown et al., 2001, as shown in Figure 4.

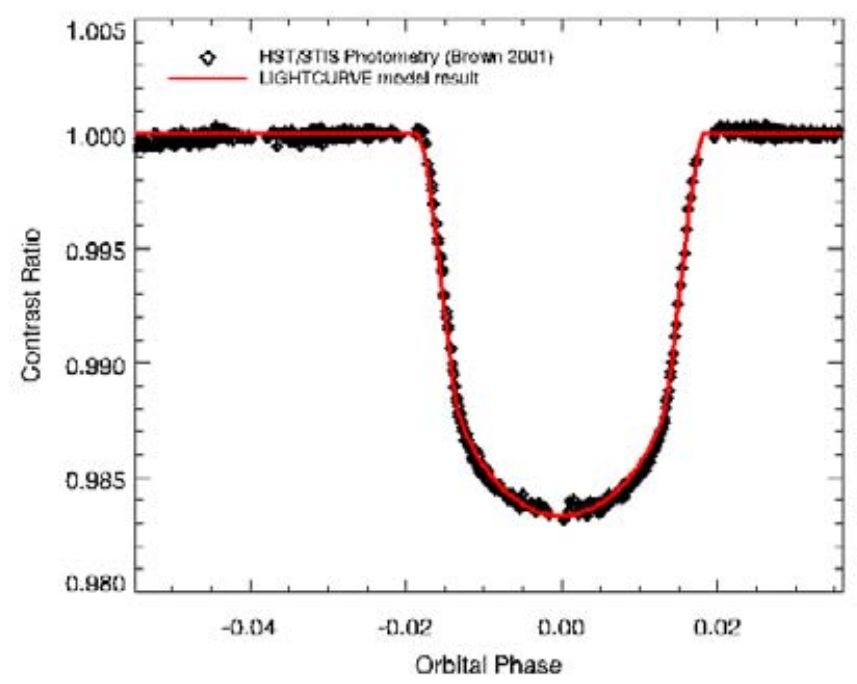

Figure 4. Points are publicly-available data from HST on the primary eclipse of HD $209458 \mathrm{~b}$ in the visible. Solid line indicates LIGHTCURVE fit for Brown et al., 2001 parameters; fit for values in eclipse (i.e., between phase of -0.02 and 0.02 ) has $\chi_{\nu}^{2}=1.969$.

\section{Predictions}

The utility of this routine for predicting the detectability of extrasolar planets is clear. Given a set of planetary and stellar parameters, we can produce a theoretical light curve,

$\dagger$ Public data can be found at http://cfa-www.harvard.edu/d̃charbon/papers.html 
and thus determine what features (i.e., temperature asymmetry, transits) may be observable with a given instrument. We integrated this routine with an instrument simulation for FKSI mission concept Danchi et al., 2003, Barry 2006 (these proceedings).

\section{Theoretical Planetary Spectra}

Here we present a brief summary of IDL routines to calculate theoretical spectra of extrasolar planetary atmospheres.

- Input temperature-pressure profile for the model atmosphere, calculated by solving the radiative transfer equation. We adopt profiles for HD 209458 b from Seager \& Sasselov, 1998 , and we compare the results to updated models to be described in a future paper (Seager, in prep, 2005).

- Tabulate line opacities for $\mathrm{H}_{2} \mathrm{O}, \mathrm{CH}_{4}$, and $\mathrm{CO}$.

- Calculate continuous opacities due to collision-induced absorption (CIA) Borysow 2002 .

- Integrate over the optical depth of the layers of the model atmosphere to obtain the emergent flux density.

\section{Future Work}

The next step in this program is to combine the spectral synthesis and light curve routines. The light curves were based on the blackbody assumption, which is clearly inadequate to describe the true nature of the planetary atmosphere. The spectral synthesis code must be expanded to include more extensive line opacity data in order to extend the calculation to $24 \mu \mathrm{m}$. We can then integrate the spectrum over a given bandpass for a particular instrument, and use this as input to the light curve routine. Finally, we will estimate noise from all sources (e.g., instrument instability, known systematics, and stellar variability), add it to the calculated light curves, and quantify to what extent the light curve is recoverable from the noisy synthetic data, thereby allowing a prediction of what features (e.g., secondary eclipse, temperature asymmetry) are detectable for the known extrasolar planets.

\section{Acknowledgements}

LJR is supported by a Research Associateship from the National Research Council at NASA Goddard.

\section{References}

Barry, R. K., et al. 2006. In C. Aime \& F. Vakili (Eds.), IAUC 200, Direct Detection of Exoplanets: Science and Techniques, in press

Borysow, A. 2002, A\& $A, 390,779$

Brown, T. M., Charbonneau, D., Gilliland, R. L., Noyes, R. W., \& Burrows, A. 2001, ApJ, 552, 699

Butler, R. P., Marcy, G. W., Williams, E., Hauser, H., \& Shirts, P. 1997, ApJ, 474, L115

Danchi, W. C., Deming, D., Kuchner, M. J., \& Seager, S. 2003, ApJ, 597, L57

Deming, D., Seager, S., Richardson, L. J., \& Harrington, J. 2005, Nature, 434, 740

Rieke, G. H., et al. 2004, ApJS, 154, 25

Seager, S., \& Sasselov, D. D. 1998, ApJ, 502, L157 